\title{
含席夫碱类配体Fe(II)配位聚合物的合成及自旋 交叉性质研究
}

仇亚茹 ${ }^{1}$, 葛景园 ${ }^{2}$, 李佳茜 ${ }^{1}$, 苏剑 $^{1 *}$, 左景林 ${ }^{1^{*}}$

1. 南京大学化学化工学院, 配位化学国家重点实验室, 南京 210023

2. 温州大学化学与材料工程学院, 温州 325035

*通讯作者, E-mail: zuoj1@nju.edu.cn

收稿日期: 2020-07-11; 接受日期: 2020-08-04; 网络版发表日期: 2020-09-14

国家重点研发计划(编号: 2018YFA0306004)、国家自然科学基金(编号: 21801054)和中国博士科学基金(编号: 2019M661788)资助项目

摘要通过常用的溶剂挥发法合成了四例含四齿席夫碱类配体的 $\mathrm{Fe}(\mathrm{II})$ 配位聚合物: [ $\left.\left(\mathrm{Fe}^{\mathrm{II}}\right)\left(\mathrm{L}_{1}\right)(4-\mathrm{abpt})\right](\mathbf{1})$ 、 $\left[\left(\mathrm{Fe}^{\mathrm{II}}\right)\left(\mathrm{L}_{2}\right)(4-\mathrm{abpt})\right](\mathbf{2}) 、\left[\left(\mathrm{Fe}^{\mathrm{II}}\right)_{2}\left(\mathrm{~L}_{3}\right)_{2}(4-\mathrm{abpt}) \cdot \mathrm{MeOH}\right] \cdot\left[\left(\mathrm{Fe}^{\mathrm{II}}\right)_{2}\left(\mathrm{~L}_{3}\right)_{2}(4-\mathrm{abpt})\right](\mathbf{3})$ 和 $\left[\left(\mathrm{Fe}^{\mathrm{II}}\right)_{3}\left(\mathrm{~L}_{4}\right)_{3}(4-\mathrm{abpt})_{2} \cdot \mathrm{H}_{2} \mathrm{O}\right] \quad(\mathbf{4})$ $\left(\mathrm{H}_{2} \mathrm{~L}_{1}=3,3^{\prime}-\left(\left(1,2\right.\right.\right.$-亚苯基双(亚胺基))双(亚甲烯))双(2,4-戊二酮), $\mathrm{H}_{2} \mathrm{~L}_{2}=$ 二乙基-2,2'-((蔡-2,3-双(亚胺基))双(亚甲烯)) (2E, $2^{\prime} E$ )-双(3-氧丁酸甲酯), $\mathrm{H}_{2} \mathrm{~L}_{3}=$ 二基-2,2'-((1,2-亚苯基双(亚胺基))双(亚甲烯))(2E,2' $E$ )-双(3-氧丁酸甲酯), $\mathrm{H}_{2} \mathrm{~L}_{4}=$ 二乙基-2,2'-(((4,5-二氟-1,2-亚苯基)双(亚胺基))双(亚甲烯))( $2 E, 2^{\prime} E$ )-双(3-氧丁酸甲酯), 4-abpt=3,5-二(吡啶4-基)-4-氢-1,2,4-三氮唑-4-胺). 对化合物 1 4 进行了元素分析、X射线粉末衍射等表征, 并利用X射线单晶衍射仪 测定了它们的单晶结构. 晶体结构分析表明, 1 4 均为一维链状结构. 利用两电极法对 1 4 进行了变温电子导电性 测试. 变温磁学性质测试结果表明, 3 呈现出一步自旋转换 $\left(T_{1 / 2}=115 \mathrm{~K}\right), 4$ 呈现两步自旋转换 $\left(T_{1 / 2}=188\right.$ 和 $\left.95 \mathrm{~K}\right)$ 的 性质.

关键词 $\mathrm{Fe}(\mathrm{II})$ 配位聚合物, 自旋交叉, 晶体结构, 席夫碱配体

\section{1 引言}

自旋交叉 $(\mathrm{SCO})$ 配合物由于可能在温度传感、光 开关和信息存储等方面得到应用而备受瞩目 ${ }^{[1 \sim 7]}$. 配合 物的自旋交叉行为包括多种不同的类型，如渐变型、 突变型、阶梯型、滞回型和不完全型，其中热滞效应 具有记忆功能 ${ }^{[8 \sim 10]}$. 研究发现, 分子间相互作用(氢 键、 $\pi \cdots \pi$ 和范德华力等)与自旋转换之间可能存在协 同作用 ${ }^{[1113]}$. 理论和实验研究表明, 协同作用越强越容
易导致滞回型的自旋转换现象. 迄今为止, 科研人员通 过修饰配体或改变溶剂、抗衡离子等方法制备出一些 具有较大热滞回温度的自旋交叉配合物 ${ }^{[9,13 \sim 15]}$. 尽管 如此, 自旋交叉体系的结构设计和磁性质调控仍然是 一大挑战. 配体设计是优化这种协同作用的有效途径 之一, 本文通过调节四齿席夫碱配体的取代基以改善 这种协同作用.

辅助桥联配体的合理选择也可以改变配合物的自 旋交叉性质. 3,5-二(吡啶-4-基)-4-氢-1,2,4-三氮唑-4-胺

引用格式: Qiu YR, Ge JY, Li JQ, Su J, Zuo JL. Spin-crossover iron(II) coordination polymers with tetradentate Schiff-base ligands. Sci Sin Chim, 2020, 50: 17371744, doi: 10.1360/SSC-2020-0128 
(4-abpt)及其衍生物是一类具有独特的 $V$ 型结构的刚性 配体. 与 $4,4^{\prime}$ - 联吡啶相比, 其具备更多的配位点. 该有 机分子及其衍生物被成功用于多例自旋交叉化合物的 组装. 例如, 2017 年, 童明良课题组 ${ }^{[16]}$ 利用 $\mathrm{Fe}\left(\mathrm{ClO}_{4}\right)_{2}{ }^{\circ}$ $6 \mathrm{H}_{2} \mathrm{O} 、 4-\mathrm{abpt}$ 和 $\mathrm{K}\left[\mathrm{Ag}(\mathrm{CN})_{2}\right]$ 合成了一例具有四步自旋 转换行为的自旋交叉配合物. 2019年，童明良课题 组 ${ }^{[17]}$ 又报道了一例新型的自旋交叉配合物 $[\mathrm{Fe}$ (dpoda) $\left.\left\{\mathrm{Ag}(\mathrm{CN})_{2}\right\}_{2}\right]$ (dpoda=2,5-二(4-吡啶基)-1,3,4-恶二唑), 磁性测试结果表明该配合物同样呈现出四步自旋转换 行为.

我们选择了 4 种结构相近的席夫碱配体 $\left(\mathrm{H}_{2} \mathrm{~L}_{1}=\right.$ 3,3'-((1,2-亚苯基双(亚胺基))双(亚甲烯))双(2,4-戊二 酮)、 $\mathrm{H}_{2} \mathrm{~L}_{2}=$ 二乙基-2,2'-((䒬-2,3-双(亚胺基))双(亚甲 烯))(2E, $\left.2^{\prime} E\right)$-双(3-氧丁酸甲酯)、 $\mathrm{H}_{2} \mathrm{~L}_{3}=$ 二乙基- $2,2^{\prime}$ $\left(\left(1,2\right.\right.$-亚苯基双(亚胺基))双(亚甲烯))( $\left.2 E, 2^{\prime} E\right)$-双 (3-氧 丁酸甲酯)、 $\mathrm{H}_{2} \mathrm{~L}_{4}=$ 二乙基- $2,2^{\prime}-(((4,5$-二氟-1,2-亚苯基 $)$ 双(亚胺基))双(亚甲烯))(2E,2'E)-双(3-氧丁酸甲酯))与 $\mathrm{V}$ 型的刚性配体4-abpt合成了 4例新颖的一维链状Fe(II) 配位聚合物(图1). 本文主要介绍了其合成、晶体结构 和磁学性质，并探讨了晶体中不同的弱相互作用对配 位聚合物的磁学性质的影响.

\section{2 实验部分}

\section{1 试剂与仪器}

实验中所用试剂未经特别说明均为化学纯或分析

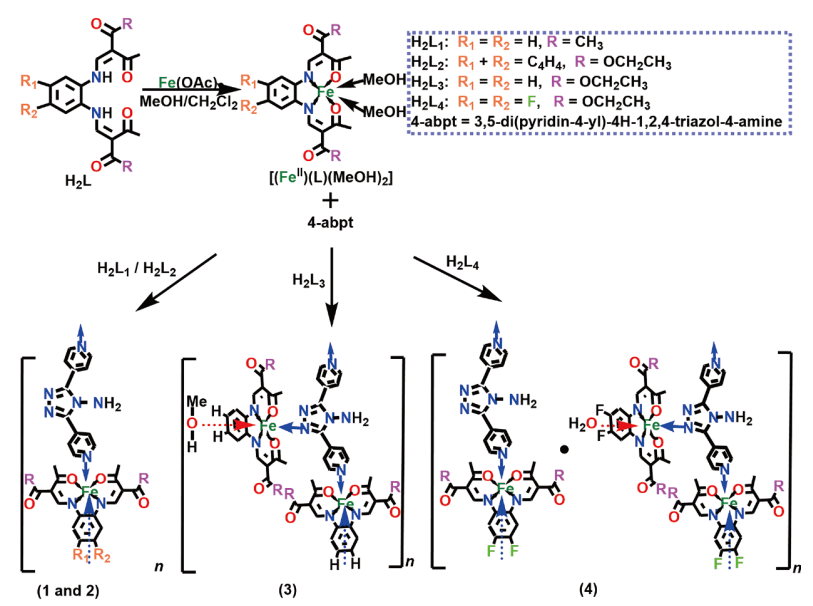

图 1 配位聚合物1 4的合成路线图(网络版彩图)

Figure 1 Synthetic pathway of spin-crossover coordination polymers 1-4 (color online).
纯, 未经进一步纯化处理. $\mathrm{H}_{2} \mathrm{~L} 、\left[\mathrm{FeL}(\mathrm{MeOH})_{2}\right]$ 和 4-abpt按照文献报道方法制备 ${ }^{[18,19]}$.

实验中所用仪器：C、H和 $\mathrm{N}$ 元素的元素分析在 Perkin-Elmer 240C元素分析仪(美国)上测得. 粉末衍 射仪器为Bruker Advance D8 (40 kV, $40 \mathrm{~mA}$, 德国), 测试为铜靶, 测试温度为室温, 扫描速率为 $0.1 \mathrm{~s} / \mathrm{step}$, 每步间隔为 $0.02^{\circ}$. 计算的粉末衍射由Mercury 3.0 软件 模拟而来. 磁性测试结果由超导量子干涉仪磁性测试 系统Quantum Design MPMS SQUID VSM获得, 所有 磁化率数据均经Pascal常数进行抗磁校正. 导电性质由 超导量子干涉仪磁性测试系统Quantum Design PPMS 测得. 粉末样品压片压力小于 1 吨, 小圆片样品由银 胶、银线联结成二电极(由于结晶溶剂的快速流失, 用 于磁性测量的都是使用完全脱溶剂的样品).

\section{2 配位聚合物 $1 \sim 4$ 的合成}

$\left[\left(\mathrm{Fe}^{\mathrm{II}}\right)\left(\mathrm{L}_{1}\right)(4-\mathrm{abpt})\right](\mathbf{1})$ 的合成: 氮气保护下, 将 $\left[\left(\mathrm{Fe}^{\mathrm{II}}\right)\left(\mathrm{L}_{1}\right)(\mathrm{MeOH})_{2}\right] \quad(50 \mathrm{mg}, 0.10 \mathrm{mmol})$ 和 $4-\mathrm{abpt}$ $(12 \mathrm{mg}, 0.05 \mathrm{mmol}$ ) 溶于 $5 \mathrm{~mL}$ 甲醇和 $5 \mathrm{~mL}$ 二氯甲烷, 室温反应过夜. 过滤, 静置, 得到红棕色棒状晶体 1 . 将 1 置于真空干燥箱 $50^{\circ} \mathrm{C}$ 干燥 $6 \mathrm{~h}$, 产率为 $24 \mathrm{mg}(70 \%$, 基 于4-abpt). 分子式为 $\mathrm{C}_{30} \mathrm{H}_{28} \mathrm{FeN}_{8} \mathrm{O}_{4}$ (分子量 $M_{\mathrm{r}}=$ $620.43 \mathrm{~g} / \mathrm{mol}$ ). 元素分析的理论结果为: C $58.08 \%, \mathrm{H}$ $4.55 \%, \mathrm{~N} 18.06 \%$; 实际实验值为: C $57.87 \%, \mathrm{H} 4.49 \%$, $\mathrm{N} 18.16 \%$.

[(Fe $\left.\left.{ }^{\mathrm{II}}\right)\left(\mathrm{L}_{2}\right)(4-\mathrm{abpt})\right](\mathbf{2})$ 的合成：合成方法与 1 类似， 用 $\left[\left(\mathrm{Fe}^{\mathrm{II}}\right)\left(\mathrm{L}_{2}\right)(\mathrm{MeOH})_{2}\right](56 \mathrm{mg}, 0.10 \mathrm{mmol})$ 替代上 述 $\left[\left(\mathrm{Fe}^{\mathrm{II}}\right)\left(\mathrm{L}_{1}\right)(\mathrm{MeOH})_{2}\right]$, 得到黑色菱形晶体 2 . 将 2 置 于真空干燥箱 $50{ }^{\circ} \mathrm{C}$ 干燥 $6 \mathrm{~h}$, 产率为 $26 \mathrm{mg}(60 \%$, 基于 4-abpt). 分子式为 $\mathrm{C}_{36} \mathrm{H}_{34} \mathrm{FeN}_{8} \mathrm{O}_{6}$ (分子量 $M_{\mathrm{r}}=$ $730.55 \mathrm{~g} / \mathrm{mol}$ ). 元素分析的理论结果为: C $59.19 \%, \mathrm{H}$ $4.69 \%, \mathrm{~N} 15.34 \%$; 实际实验值为: C 58.98\%, H 4.55\%, N $15.42 \%$.

$\left[\left(\mathrm{Fe}^{\mathrm{II}}\right)_{2}\left(\mathrm{~L}_{3}\right)_{2}(4-\mathrm{abpt}) \cdot \mathrm{MeOH}\right] \cdot\left[\left(\mathrm{Fe}^{\mathrm{II}}\right)_{2}\left(\mathrm{~L}_{3}\right)(4-\mathrm{abpt})\right](\mathbf{3})$ 的合成: 合成方法与 $\mathbf{1}$ 类似, 用 $\left[\left(\mathrm{Fe}^{\mathrm{II}}\right)\left(\mathrm{L}_{3}\right)(\mathrm{MeOH})_{2}\right]$ $(51 \mathrm{mg}, 0.10 \mathrm{mmol})$ 替代上述 $\left[\left(\mathrm{Fe}^{\mathrm{II}}\right)\left(\mathrm{L}_{1}\right)(\mathrm{MeOH})_{2}\right]$, 得到 黑色菱形晶体3. 将 3 置于真空干燥箱 $50^{\circ} \mathrm{C}$ 干燥 $6 \mathrm{~h}$, 产率 为 $81 \mathrm{mg}\left(65 \%\right.$, 基于4-abpt). 分子式为 $\mathrm{C}_{106} \mathrm{H}_{116} \mathrm{Fe}_{4} \mathrm{~N}_{20} \mathrm{O}_{26}$ (分子量 $M_{\mathrm{r}}=2309.55 \mathrm{~g} / \mathrm{mol}$ ). 元素分析的理论结果为: C $55.12 \%, \mathrm{H} 5.06 \%, \mathrm{~N} 12.13 \%$; 实际实验值为: $\mathrm{C}$ $54.91 \%$, H $5.01 \%$, N $12.12 \%$. 
$\left[\left(\mathrm{Fe}^{\mathrm{II}}\right)_{3}\left(\mathrm{~L}_{4}\right)_{3}(4-\mathrm{abpt})_{2} \cdot \mathrm{H}_{2} \mathrm{O}\right](4)$ 的合成: 合成方法与 1类似，用 $\left[\left(\mathrm{Fe}^{\mathrm{II}}\right)\left(\mathrm{L}_{4}\right)(\mathrm{MeOH})_{2}\right](54 \mathrm{mg}, 0.10 \mathrm{mmol})$ 替代 上述 $\left[\left(\mathrm{Fe}^{\mathrm{II}}\right)\left(\mathrm{L}_{1}\right)(\mathrm{MeOH})_{2}\right]$, 得到黑色菱形晶体 4 . 将 4 置 于真空干燥箱 $50^{\circ} \mathrm{C}$ 干燥 $10 \mathrm{~h}$, 产率为 $65 \mathrm{mg}(65 \%$, 基于 4-abpt). 分子式为 $\mathrm{C}_{84} \mathrm{H}_{82} \mathrm{~F}_{6} \mathrm{Fe}_{3} \mathrm{~N}_{18} \mathrm{O}_{19}$ (分子量 $M_{\mathrm{r}}=$ $1929.19 \mathrm{~g} / \mathrm{mol})$. 元素分析的理论结果为: C $52.30 \%, \mathrm{H}$ $4.28 \%, \mathrm{~N} 13.07 \%$; 实际实验值为: C $52.08 \%, \mathrm{H} 4.19 \%$, N $13.15 \%$.

\section{3 结果与讨论}

\section{1 配位聚合物 1 4 的结构分析}

配位聚合物 1 4 的晶体学参数列于表 $\mathrm{S} 1$ 和 $\mathrm{S} 2$ (网 络版补充材料), 主要键长和键角列于表 $\mathrm{S} 3 \sim \mathrm{S} 6$.

$100 \mathrm{~K}$ 时，1结晶属于单斜晶系，属于 $P 2_{1} / c$ 空间群. 其最小不对称单元包含一个 Fe(II)、一个席夫碱配体 和一个4-abpt配体(图2(a)). 中心 $\mathrm{Fe}(\mathrm{II})$ 处于 $\left\{\mathrm{FeN}_{4} \mathrm{O}_{2}\right\}$ 的
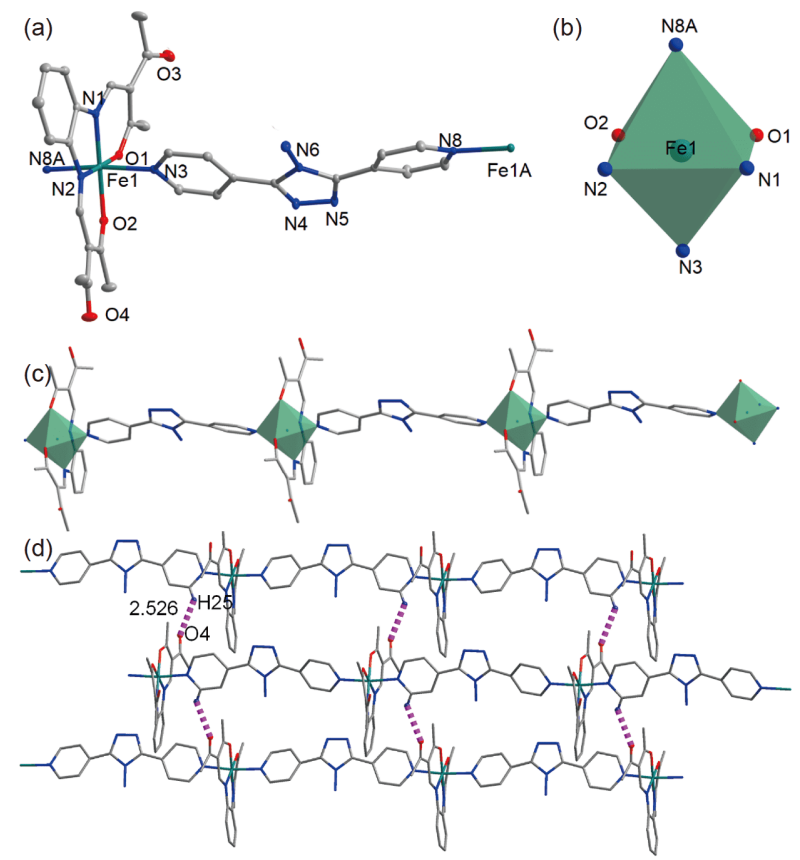

图 2 (a) 1 的晶体结构; (b) 金属中心Fe(II)离子的配位环境; (c)一维链状结构图; (d) 二维网状结构图(为清晰可见, 非必 要氢原子已被略去，碳原子-灰色，氮原子-蓝色，氧原子-红 色, 铁原子-青色) (网络版彩图)

Figure 2 (a) The crystal structure of 1; (b) coordination environment of central Fe(II); 1D chain (c) and 2D network (d) of 1 (All of the unnecessary hydrogen atoms are omitted for clarity, carbon-gray, nitrogen-blue, oxygen-red, iron-teal) (color online).
八面体配位环境中, 席夫碱配体 $(\mathrm{N} 1 、 \mathrm{~N} 2 、 \mathrm{O} 1$ 和 $\mathrm{O} 2)$ 位 于赤道平面, 两个吡啶 $\mathrm{N}$ 原子 $(\mathrm{N} 3$ 和 $\mathrm{N} 8 \mathrm{~A})$ 占据八面体的 轴向位置(图2(b)). Fe1-O和Fe1-N的平均键长分别为 1.937 和 $1.943 \AA$, 键角 $\mathrm{O} 1-\mathrm{Fe} 1-\mathrm{O} 2$ 是 $88.34^{\circ}$, 它们与文 献报道的低自旋 $\mathrm{Fe}(\mathrm{II})$ 离子的键长和键角数值相

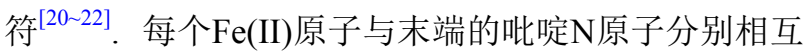
连接形成…Fe-py-Fe-py…交替的一维链状结构(图2 (c)). 此外, 相邻的一维链状结构通过分子间的弱作用 $\left(\mathrm{C} 25-\mathrm{H} 25 \cdots \mathrm{O} 4: 2.526 \AA, 135.58^{\circ}\right)$ 相互连接形成二维 网状结构(图2(d)).

配合物 $\mathbf{2}$ 的配位环境和自组装空间结构与 $\mathbf{1}$ 相似 (图S1(b), 网络版补充材料), 相邻的一维链状结构通过 分子间的弱作用(N7-H7A ‥O6: $2.119 \AA, 141.50^{\circ}$ )相互 连接形成二维网状结构(图3(a)). 此外, 通过分子间的 弱作用 $(\mathrm{C} 17-\mathrm{H} 17 \mathrm{C} \cdots \pi$ (py ring: C32, C33, C34, C35, C36, N8): $3.813 \AA, 171.49^{\circ}$ )二维网状结构可进一步构 筑成三维结构(图3(b)).

配合物 3 中有两个单体(分子 $\mathrm{A}$ 和分子 $\mathrm{B}$ ), 除了键长 和键角的微小差异, 两个独立分子的配位模式是一样 的(图4(a)). 因此，我们选择A分子来详细描述. 分子A 由两个独立的 $\mathrm{Fe}(\mathrm{II})$ 离子、一个席夫碱配体和一个4abpt配体组成, 每一个中心 $\mathrm{Fe}(\mathrm{II})$ 都是六配位, 呈微微扭 曲的八面体构型(图4(b)). 在 $100 \mathrm{~K}$ 时, 3 的中心原子 $\mathrm{Fe} 1$ : 席夫碱配体中的两个氮原子和两个氧原子占据 了赤道位置 $\left(\mathrm{O}_{\mathrm{eq}}-\mathrm{Fe}-\mathrm{O}_{\mathrm{eq}}\right.$ 角: $\left.\mathrm{O} 1-\mathrm{Fe} 1-\mathrm{O} 490.52^{\circ}\right)$, 4-abpt

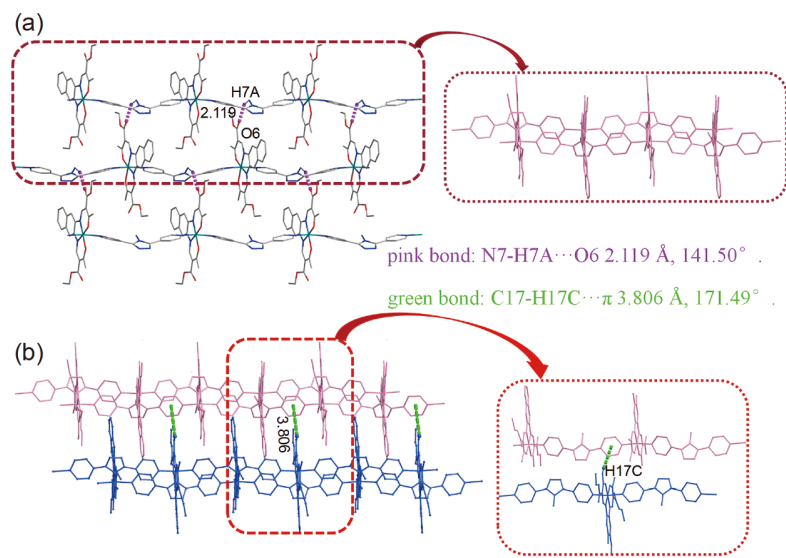

图 3 (a) 2 的二维网状结构; (b) 三维结构图(为清晰可见, 非 必要氢原子已被略去, 碳原子-灰色, 氮原子-蓝色, 氧原子-红 色, 铁原子-青色) (网络版彩图)

Figure 3 The 2D network (a) and 3D structure (b) of 2 (All of the unnecessary hydrogen atoms are omitted for clarity, carbon-gray, nitrogen-blue, oxygen-red, iron-teal) (color online). 

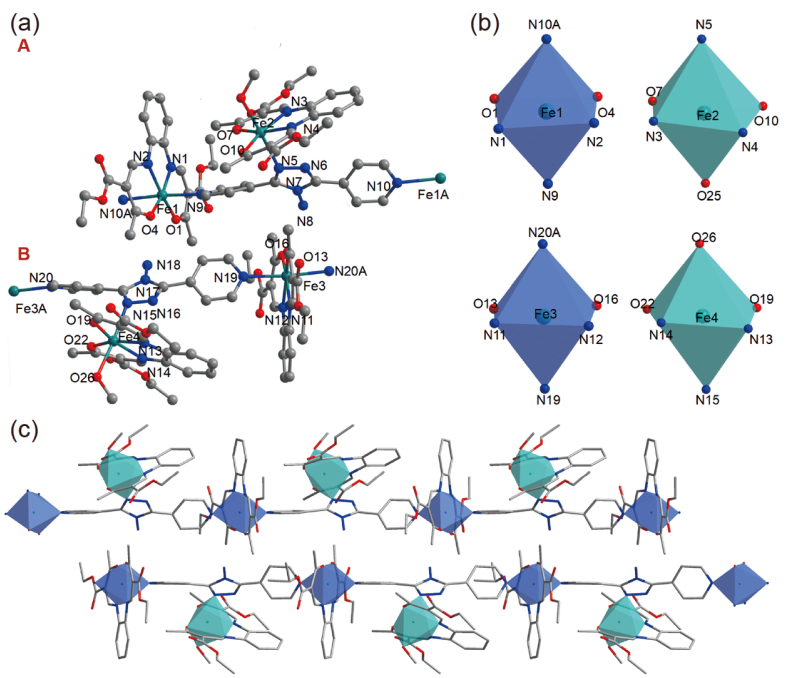

图 4 (a) 3 的晶体结构; (b) 金属中心Fe(II)离子的配位环境; (c) 一维链状结构图(为清晰可见, 氢原子已被略去, 碳原子灰色，氮原子-蓝色，氧原子-红色，铁原子-青色) (网络版彩 图)

Figure 4 The crystal structure (a) and coordination environment of independent central Fe(II) (b) and 1D chain (c) of 3 (All of the hydrogen atoms are omitted for clarity, carbon-gray, nitrogen-blue, oxygen-red, iron-teal) (color online).

桥联配体中的两个氮原子占据了轴向位置(轴向角: N9-Fe1-N10A $\left.176.85^{\circ}\right)$, 中心原子Fe2：赤道位置也是 被来自于席夫碱配体的两个氮原子和两个氧原子占据 着 $\left(\mathrm{O}_{\mathrm{eq}}-\mathrm{Fe}-\mathrm{O}_{\mathrm{eq}}\right.$ 角: $\left.\mathrm{O} 7-\mathrm{Fe} 2-\mathrm{O} 10109.73^{\circ}\right)$, 轴向位置被 一个来自于配位甲醇的氧原子和一个来自于 4-abpt配 体的氮原子占据(N5-Fe2-O25 176.22 $)$. 中心原子 Fe1 与 $\mathrm{Fe} 3$ 的配位环境相似, 轴向角: $\mathrm{N} 19-\mathrm{Fe} 3-\mathrm{N} 20 \mathrm{~B}$ $175.65^{\circ} ; \mathrm{O}_{\mathrm{eq}}-\mathrm{Fe}-\mathrm{O}_{\mathrm{eq}}$ 角: $\mathrm{O} 13-\mathrm{Fe} 3-\mathrm{O} 16$ 91.89 ; 中心原 子 $\mathrm{Fe} 4$ 与 $\mathrm{Fe} 2$ 的配位环境相似，轴向角： N15-Fe4-O26 $174.21^{\circ} ; \mathrm{O}_{\mathrm{eq}}-\mathrm{Fe}-\mathrm{O}_{\mathrm{eq}}$ 角: $\mathrm{O} 19-\mathrm{Fe} 4-\mathrm{O} 22111.90^{\circ}$. 从 $\mathrm{O}_{\mathrm{eq}}-$ $\mathrm{Fe}-\mathrm{O}_{\mathrm{eq}}$ 角的大小可知, $\mathrm{Fe} 1 、 \mathrm{Fe} 3$ 与文献报道的低自旋 $\mathrm{Fe}(\mathrm{II})$ 离子的键长、键角一致, 而 $\mathrm{Fe} 2$ 与 $\mathrm{Fe} 4$ 都处于高自 旋(表1) ${ }^{[21,22]}$. 此外, 对中心原子 $\mathrm{Fe} 1$ 与 $\mathrm{Fe} 3: \mathrm{Fe}-\mathrm{O}$ 和 $\mathrm{Fe}-\mathrm{N}$ 的平均键长分别为 $1.944 、 1.970 \AA$ 和 $1.949 、 1.951 \AA$, 对中心原子 $\mathrm{Fe} 2$ 与 $\mathrm{Fe} 4: \mathrm{Fe}-\mathrm{O}$ 和 $\mathrm{Fe}-\mathrm{N}$ 的平均键长分别为 $2.065 、 2.152 \AA$ 和 $2.069 、 2.155 \AA$, 这些键长数据也可 以证实中心原子 $\mathrm{Fe} 1$ 与 $\mathrm{Fe} 3$ 处于低自旋，中心原子 $\mathrm{Fe} 2$ 与 $\mathrm{Fe} 4$ 处于高自旋 $(\text { 表 } 1)^{[20-22]}$. 当温度升至 $250 \mathrm{~K}$, 所有 键长都变得更长, 平均键长 $d(\mathrm{Fe}-\mathrm{N})=2.175 \AA, d(\mathrm{Fe}-\mathrm{O})$ $=2.53 \AA$; 所有的键角都变得更大, $\mathrm{Fe} 1 、 \mathrm{Fe} 2 、 \mathrm{Fe} 3$ 和 $\mathrm{Fe} 4$ 的 $\mathrm{O}_{\mathrm{eq}}-\mathrm{Fe}-\mathrm{O}_{\mathrm{eq}}$ 角度分别为 $106.90^{\circ} 、 109.20^{\circ} 、 108.60^{\circ}$ 和
表 1 配位聚合物 $\mathbf{3}$ 的中心 $\mathrm{Fe}(\mathrm{II})$ 原子的平均键长 $(\AA)$ 和键角 $\left(^{\circ}\right.$ )列表

Table 1 The average bond lengths $(\AA)$ and angles $\left({ }^{\circ}\right)$ of central Fe(II) in coordination polymer $\mathbf{3}$

\begin{tabular}{ccc}
\hline $\mathbf{3}$ & $100 \mathrm{~K}$ & $250 \mathrm{~K}$ \\
\hline $\mathrm{Fe} 1-\mathrm{O}$ & $1.944 \AA$ & $2.020 \AA$ \\
$\mathrm{Fe} 1-\mathrm{N}$ & $1.970 \AA$ & $2.174 \AA$ \\
$\mathrm{O} 1-\mathrm{Fe} 1-\mathrm{O} 4$ & $90.52^{\circ}$ & $106.90^{\circ}$ \\
$\mathrm{Fe} 2-\mathrm{O}$ & $2.065 \AA$ & $2.084 \AA$ \\
$\mathrm{Fe} 2-\mathrm{N}$ & $2.152 \AA$ & $2.166 \AA$ \\
$\mathrm{O} 7-\mathrm{Fe} 2-\mathrm{O} 10$ & $109.73^{\circ}$ & $109.20^{\circ}$ \\
$\mathrm{Fe} 3-\mathrm{O}$ & $1.949 \AA$ & $2.020 \AA$ \\
$\mathrm{Fe} 3-\mathrm{N}$ & $1.951 \AA$ & $2.189 \AA$ \\
$\mathrm{O} 13-\mathrm{Fe} 3-\mathrm{O} 16$ & $91.89^{\circ}$ & $108.6^{\circ}$ \\
$\mathrm{Fe} 4-\mathrm{O}$ & $2.069 \AA$ & $2.087 \AA$ \\
$\mathrm{Fe} 4-\mathrm{N}$ & $2.155 \AA$ & $2.170 \AA$ \\
$\mathrm{O} 19-\mathrm{Fe} 4-\mathrm{O} 22$ & $111.90^{\circ}$ & $111.0^{\circ}$ \\
\hline
\end{tabular}

$111.00^{\circ}$, 这些晶体数据都说明 $\mathrm{Fe} 1 、 \mathrm{Fe} 2 、 \mathrm{Fe} 3$ 和 $\mathrm{Fe} 4$ 均 处于高自旋. 对于中心原子 $\mathrm{Fe} 1$, 每个 Fe(II) 原子与末端 的吡啶 $\mathrm{N}$ 原子分别相互连接形成 $\cdots \mathrm{Fe}-\mathrm{py}-\mathrm{Fe}-\mathrm{py} \cdots$ 交 替的一维链状结构(图4(c)). 相邻的一维链状结构通过 分子间的弱作用 $\left(\mathrm{C} 51-\mathrm{H} 51 \cdots \mathrm{O} 12: 2.490 \AA, 159.71^{\circ}\right)$ 相 互连接形成二维网状结构(图S2). 单晶结构分析表明3 存在热诱导自旋转换性质.

配位聚合物 4 也有两个单体(分子 $\mathrm{A}$ 和分子 $\mathrm{B}$ )。其 中, 分子 $\mathrm{A}$ 的中心铁原子 $(\mathrm{Fe} 1)$ 的配位环境和 1 的相似, 而分子 $\mathrm{B}$ 的中心铁原子 $(\mathrm{Fe} 2$ 和 $\mathrm{Fe} 3)$ 的配位环境和 3 的相 似(图5(a, b)). 在 $100 \mathrm{~K}, 4$ 的平均键长Fe1-O $1.944 \AA$, Fe1-N $1.954 \AA$, Fe2-O $2.107 \AA$, Fe2-N 2.134 Å, Fe3-O $1.948 \AA, \mathrm{Fe} 3-\mathrm{N} 1.951 \AA$; 键角 $\mathrm{O}_{\mathrm{eq}}-\mathrm{Fe}-\mathrm{O}_{\text {eq }}$ : O3-Fe1-O6 $89.58^{\circ}, \mathrm{O} 9-\mathrm{Fe} 2-\mathrm{O} 12 \quad 109.68^{\circ}$ 和O16-Fe3-O19 $90.54^{\circ}$ (表2). 基于这些晶体参数, Fe1和Fe3处于低自旋, 而 $\mathrm{Fe} 2$ 处于高自旋. 随着温度的升高, 键长变得越来越长, 键角变得越来越大，通过相应的键长、键角的变化可 以准确地判断中心金属原子 $\mathrm{Fe}$ 的高低自旋状态 ${ }^{[20]}$. 当 温度升至 $160 \mathrm{~K}$, 平均键长 $d(\mathrm{Fe}-\mathrm{N})=2.031 \AA, d(\mathrm{Fe}-\mathrm{O})$ $=2.002 \AA$; 所有的键角 $\mathrm{Fe} 1 、 \mathrm{Fe} 2$ 和 $\mathrm{Fe} 3$ 的 $\mathrm{O}_{\mathrm{eq}}-\mathrm{Fe}-\mathrm{O}_{\mathrm{eq}}$ 角 分别为 $92.36^{\circ} 、 110.26^{\circ}$ 和 $91.59^{\circ}$; 当温度升至 $250 \mathrm{~K}$, 平均键长 $d(\mathrm{Fe}-\mathrm{O})=2.046 \AA, d(\mathrm{Fe}-\mathrm{N})=2.161 \AA$, 所有的 键角 $\mathrm{Fe} 1 、 \mathrm{Fe} 2$ 和 $\mathrm{Fe} 3$ 的 $\mathrm{O}_{\mathrm{eq}}-\mathrm{Fe}-\mathrm{O}_{\mathrm{eq}}$ 角分别为 $108.58^{\circ}$ 、 $107.98^{\circ}$ 和 $106.74^{\circ}$; 此时 $\mathrm{Fe} 1 、 \mathrm{Fe} 2$ 和 $\mathrm{Fe} 3$ 均处于高自旋; 
(a) $\mathrm{A}$
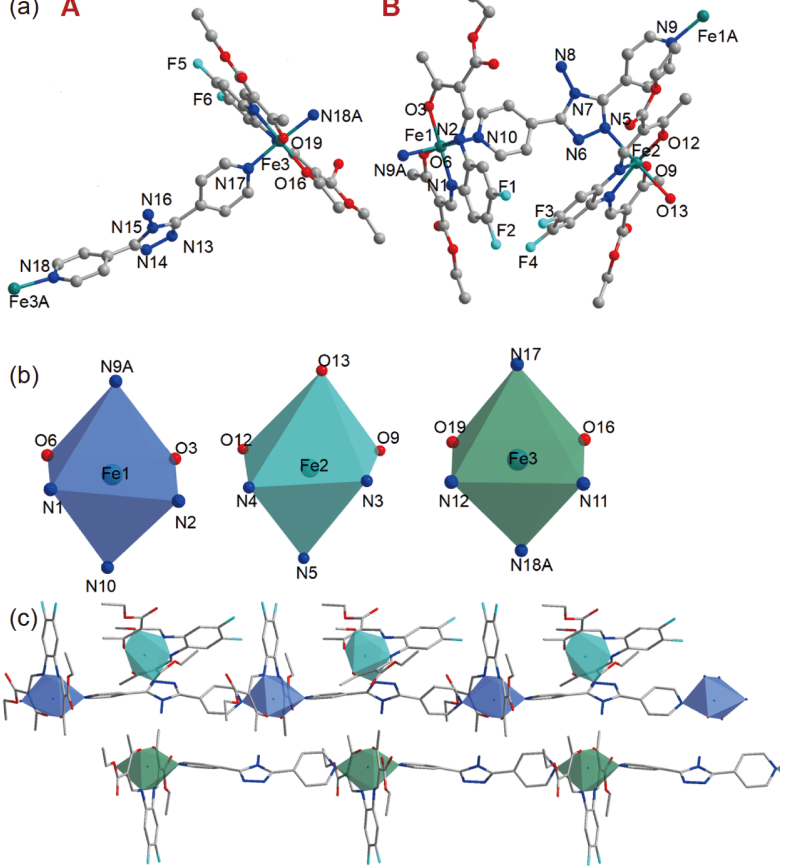

图 5 (a) 4的晶体结构; (b) 金属中心Fe(II)离子的配位环境; (c) 一维链状结构图(为清晰可见, 氢原子已被略去, 碳原子灰色, 氮原子-蓝色, 氧原子-红色, 铁原子-青色) (网络版 彩图)

Figure 5 The crystal structure (a) and coordination environment of independent central $\mathrm{Fe}(\mathrm{II})$ (b) and 1D chains (c) of 4 (All of the hydrogen atoms are omitted for clarity, carbon-gray, nitrogen-blue, oxygen-red, iron-teal) (color online).

表 2 配位聚合物 $\mathbf{4}$ 的中心 $\mathrm{Fe}(\mathrm{II})$ 原子的平均键长 $(\AA)$ 和键角 ${ }^{\circ}$ )列表

Table 2 The average bond lengths $(\AA)$ and angles $\left({ }^{\circ}\right)$ of central Fe(II) in coordination polymer $\mathbf{4}$

\begin{tabular}{ccccc}
\hline 4 & $100 \mathrm{~K}$ & $160 \mathrm{~K}$ & $250 \mathrm{~K}$ & $298 \mathrm{~K}$ \\
\hline $\mathrm{Fe} 1-\mathrm{O}$ & $1.944 \AA$ & $1.951 \AA$ & $2.007 \AA$ & $1.992 \AA$ \\
$\mathrm{Fe} 1-\mathrm{N}$ & $1.954 \AA$ & $1.978 \AA$ & $2.186 \AA$ & $2.189 \AA$ \\
$\mathrm{O} 3-\mathrm{Fe} 1-\mathrm{O} 6$ & $89.58^{\circ}$ & $92.36^{\circ}$ & $108.58^{\circ}$ & $107.1^{\circ}$ \\
$\mathrm{Fe} 2-\mathrm{O}$ & $2.107 \AA$ & $2.103 \AA$ & $2.127 \AA$ & $2.101 \AA$ \\
$\mathrm{Fe} 2-\mathrm{N}$ & $2.134 \AA$ & $2.148 \AA$ & $2.129 \AA$ & $2.119 \AA$ \\
$\mathrm{O} 9-\mathrm{Fe} 2-\mathrm{O} 12$ & $109.68^{\circ}$ & $110.26^{\circ}$ & $107.98^{\circ}$ & $108.10^{\circ}$ \\
$\mathrm{Fe} 3-\mathrm{O}$ & $1.948 \AA$ & $1.951 \AA$ & $2.003 \AA$ & $2.014 \AA$ \\
$\mathrm{Fe} 3-\mathrm{N}$ & $1.951 \AA$ & $1.968 \AA$ & $2.167 \AA$ & $2.162 \AA$ \\
$\mathrm{O} 16-\mathrm{Fe} 3-\mathrm{O} 19$ & $90.54^{\circ}$ & $91.59^{\circ}$ & $106.74^{\circ}$ & $109.0^{\circ}$ \\
\hline
\end{tabular}

同样地, 当温度升至 $298 \mathrm{~K}$, 平均键长 $d(\mathrm{Fe}-\mathrm{O})=2.035 \AA$, $d(\mathrm{Fe}-\mathrm{N})=2.157 \AA$, 所有的键角 $\mathrm{Fe} 1 、 \mathrm{Fe} 2$ 和 $\mathrm{Fe} 3$ 的
$\mathrm{O}_{\mathrm{eq}}-\mathrm{Fe}-\mathrm{O}_{\mathrm{eq}}$ 角分别为 $107.1^{\circ} 、 108.1^{\circ}$ 和 $109.0^{\circ}$; 此时的 $\mathrm{Fe} 1 、 \mathrm{Fe} 2$ 和Fe3均处于高自旋 ${ }^{[20-22]}$. 对于中心原子 Fe1 和 $\mathrm{Fe} 3$ ，每个 $\mathrm{Fe}(\mathrm{II})$ 原子与末端的吡啶 $\mathrm{N}$ 原子分别相互 连接形成 $\cdots \mathrm{Fe}-\mathrm{py}-\mathrm{Fe}-\mathrm{py} \cdots$ 交替的一维链状结构(图5 (c)). 和 3 一样, 4 中也发生了热诱导的自旋转换行为.

\section{$3.2 \mathrm{X}$ 射线粉末衍射}

如图S6所示，配位聚合物 1 4 的粉末X射线衍射花 样与单晶结构模拟的结果基本一致，说明它们的多晶 状态同单晶结构一致, 并且不含其它杂质相.

\section{3 磁学性质}

在温度为300 2 K、外加磁场 $1 \mathrm{kOe}$ 下，对配位聚 合物1 4的变温磁化率进行了测试. 此外，我们也对 3 和4从2 300 K的升温曲线进行了测试, 升温与降温曲 线重合, 并没有观察到热滞回现象(图6).

在测试的整个温度区间内, 1 始终处于低自旋的状 态，室温 $(300 \mathrm{~K})$ 下， 1 的摩尔磁化率 $\chi_{\mathrm{M}} T$ 实验值近似为 $0.06 \mathrm{~cm}^{3} \mathrm{~K} \mathrm{~mol}^{-1}$. 这一结果与 $\mathrm{X}$ 射线单晶结构分析的 结果一致. 室温 $(300 \mathrm{~K})$ 下, 2 的摩尔磁化率 $\chi_{\mathrm{M}} T$ 实验值 为 $3.28 \mathrm{~cm}^{3} \mathrm{~K} \mathrm{~mol}^{-1}$, 与一个高自旋态 $\mathrm{Fe}(\mathrm{II})$ 离子的理论 值吻合 ${ }^{[20 \sim 22]}$. 在300 30 K, $\chi_{\mathrm{M}} T$ 值逐渐缓慢地减小, 在 $30 \mathrm{~K}$ 时达 $3.03 \mathrm{~cm}^{3} \mathrm{~K} \mathrm{~mol}^{-1}$. 随后在 $30 \mathrm{~K}$ 以下, $\chi_{\mathrm{M}} T$ 值迅 速减小, 在 $2 \mathrm{~K}$ 时达 $2.22 \mathrm{~cm}^{3} \mathrm{~K} \mathrm{~mol}^{-1}$, 可能是高自旋态 $\mathrm{Fe}(\mathrm{II})$ 离子的零场分裂导致的. $300 \mathrm{~K}$ 时, 3 的摩尔磁化
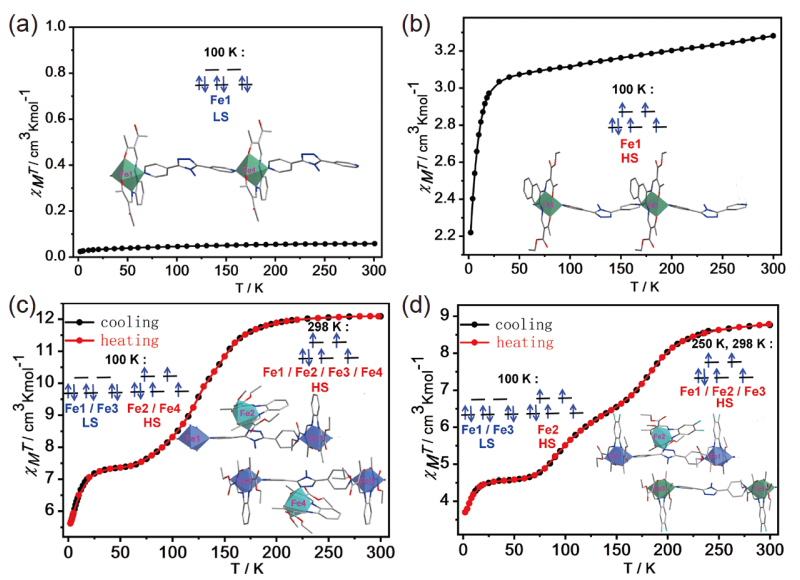

图 6 温度2 300 K、外加磁场 $1 \mathrm{kOe}, \mathbf{1} \sim \mathbf{4}(\mathrm{a} \sim \mathrm{d})$ 的变温磁 化率曲线(网络版彩图)

Figure 6 Temperature dependence of $\chi_{\mathrm{M}} T$ data in warming and cooling modes for 1-4 (a-d) under $1 \mathrm{kOe}$ applied dc field and 2-300 K (color online). 
率 $\chi_{\mathrm{M}} T$ 实验值为 $12.09 \mathrm{~cm}^{3} \mathrm{~K} \mathrm{~mol}^{-1}$, 这说明每一个 $\mathrm{Fe}(\mathrm{II})$ 都处于高自旋态. 在 $300 \sim 180 \mathrm{~K}, \chi_{\mathrm{M}} T$ 从 12.09 降为 $11.68 \mathrm{~cm}^{3} \mathrm{~K} \mathrm{~mol}^{-1}$, 在180 65 K, $\chi_{\mathrm{M}} T$ 迅速减小, 然后再 缓慢减小, 直到 $25 \mathrm{~K}, \chi_{\mathrm{M}} T$ 减小为 $7.18 \mathrm{~cm}^{3} \mathrm{~K} \mathrm{~mol}^{-1}$, 这 表明 3 具有渐变且不完全的自旋交叉行为. 随后在 $25 \mathrm{~K}$ 以下, $\chi_{\mathrm{M}} T$ 迅速下降, 这可能是由残留高自旋态 $\mathrm{Fe}(\mathrm{II})$ 离 子的零场分裂导致的, 而不是来自分子间的反铁磁相 互作用 (由于分子内 $\mathrm{Fe} \cdots \mathrm{Fe}$ 最短距离为 $7.543 \AA$, 金属 离子之间的相互作用很弱 $)^{[8]}$. 3 的降温和升温测试曲 线重合, 自旋转换温度 $T_{1 / 2}$ 为 $115 \mathrm{~K}$. 室温下, 4 的 $\chi_{\mathrm{M}} T$ 值为 $8.76 \mathrm{~cm}^{3} \mathrm{~K} \mathrm{~mol}^{-1}$, 在 $300 \sim 2 \mathrm{~K}$ 之间包含两步自 旋转换. 第一步自旋转换:在300 165 K区间, $\chi_{\mathrm{M}} T$ 值呈 先慢后快的降低趋势, 在 $165 \mathrm{~K}$ 达 $6.86 \mathrm{~cm}^{3} \mathrm{~K} \mathrm{~mol}^{-1}$, 自 旋转换的温度 $T_{1 / 2}$ 为 $188 \mathrm{~K}$. 第二步自旋转换：温度低 于 $165 \mathrm{~K}, \chi_{\mathrm{M}} T$ 值仍然降低, 先是迅速降低, 然后逐渐缓 慢降低，在 $20 \mathrm{~K}$ 时达 $4.46 \mathrm{~cm}^{3} \mathrm{~K} \mathrm{~mol}^{-1}$. 这一步的自旋 转换温度 $T_{1 / 2}$ 为 $95 \mathrm{~K}$, 此时依然约有 $50 \%$ 的 $\mathrm{Fe}(\mathrm{II})$ 离 子处于高自旋态，表明 4 具有渐变且不完全的自旋 交叉行为. $20 \mathrm{~K}$ 以下, $\chi_{\mathrm{M}} T$ 迅速下降, $2 \mathrm{~K}$ 时, $\chi_{\mathrm{M}} T$ 为 $3.70 \mathrm{~cm}^{3} \mathrm{~K} \mathrm{~mol}^{-1}$.

配位聚合物 1 4 的磁学性质与 X射线衍射单晶结 构特征基本一致. 这一系列的数据证实了晶体结构和 磁学性质之间的强相关性. 取代基的不同会导致铁中 心配体场强度的差异, 配合物中存在两个或两个以上 的不等价的铁中心会引起阶梯式自旋跃迁 ${ }^{[23]}$. 此外, 协同作用的数量以及强度对配合物的自旋交叉性质有 一定的影响 ${ }^{[22,24]}$. 配位聚合物 $\mathbf{1}$ 代表了理想的一维链状 结构, $\mathbf{1}$ 在磁性测量的温度范围内处于低自旋. 配位聚 合物 $\mathbf{2}$ 表现出顺磁性, 这很可能是由于配体基团的微小 变化改变了中心 $\mathrm{Fe}(\mathrm{II})$ 原子的配体场整体强度, 从而导 致 1 和 2 的磁性有所差异. 而分子间相互作用的研究对 理解磁性质具有重要意义. 尤其是相互作用间的差异 极易导致配合物的磁性差异. 在配位聚合物 $\mathbf{3}$ 和 4 中, 不仅存在多个不对称的铁中心，还具有多种强度不同 的氢键作用(表S7和图S4, S5). 对于 3 , “之”字链结构的 一维链和链与链之间的氢键作用, 使铁中心呈现出一 步自旋转换现象 ${ }^{[25,26]}$. 对于 4 , 一维链状的“之”字链结 构、链与链之间的氢键作用以及分子内的经典氢键作 用, 使铁中心呈现出两步自旋转换现象 ${ }^{[25,26]}$. 此外, $\mathrm{Fe}(\mathrm{II})$ 中心的整体配体场强度可能是它们自旋交叉特 性不同的一个关键因素.

\section{4 变温电子导电性质}

配位聚合物 1 4 压片样品(薄片的厚度为 $0.1 \mathrm{~cm}$, 表面积为 $0.1256 \mathrm{~cm}^{2}$ ) 的变温电阻率的测量条件和方法 是: 零场下, 在300 2 K温度范围内使用双电极法测试 获得(图7).

室温下, $1 \sim 4$ 的电阻率分别为 $4.409 \times 10^{7}$ 、 $4.593 \times 10^{7} 、 4.609 \times 10^{7}$ 和 $4.414 \times 10^{7} \Omega \mathrm{cm}$. 变温电阻率 曲线表明, 在测试的温度范围内, 随着温度的降低, 电 阻率逐渐降低. 3和 4 的电阻率分别在 265 185 K和 265 75 K的温度范围内明显降低, 其变化趋势与自旋 转变相吻合(图7), 这表明配位聚合物的磁学性质和导 电性质之间存在着某种相互作用. 配位聚合物 $\mathbf{3}$ 和 $\mathbf{4}$ 的 变温晶体测试表明, 键长、键角和Fe(II)高、低自旋态 的改变会导致配位聚合物的电导率也发生相应的变 化. 与自旋交叉相关的几何和电子因素以及相互作用 需要全面考虑, 才可能理解结构与性质、导电和磁性 之间的关系 ${ }^{[24]}$.

\section{4 结论}

本文以 4 种结构相近的四齿席夫碱配体, 分别与 $\mathrm{Fe}(\mathrm{OAc})_{2}$ 和桥联配体4-abpt结合得到了 4例 Fe(II)配位 聚合物，并对其进行了X射线单晶衍射、元素分析、 磁性以及导电等一系列表征. 结果表明, 配位聚合物 1 4 均是一维链状结构; 1 处于低自旋, 2 是顺磁性的材 料, 3 呈现一步自旋转换, 4 呈现两步自旋转换. 不同类
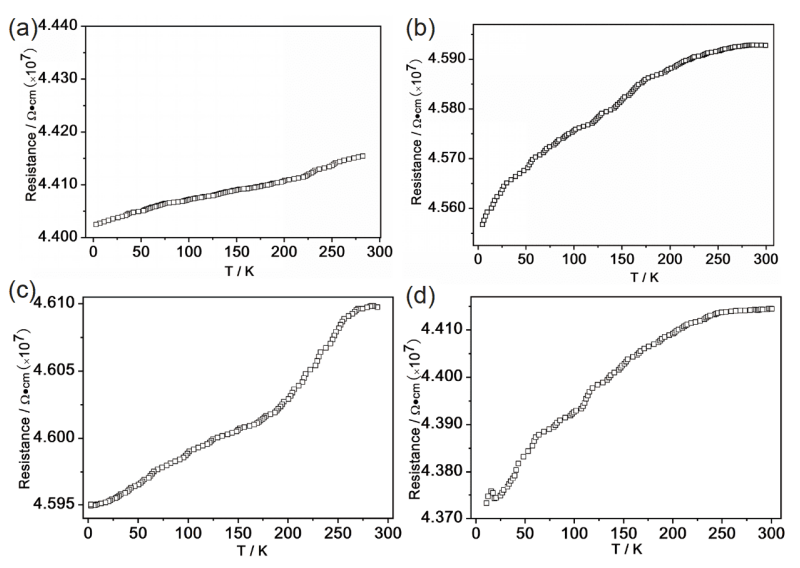

图 7 温度 $2 \sim 300 \mathrm{~K}$ 、零场下, $1 \sim 4 \quad(\mathrm{a} \sim \mathrm{d})$ 的变温电子导电 测试

Figure 7 Temperature dependence of electrical data 1-4 (a-d) under zero de field and $2-300 \mathrm{~K}$. 
型的磁作用归因于它们结构上的微小差异, 结构中多 个不对称铁中心的存在使得配合物呈现多步自旋转
变. 1 4 的变温电导率的变化与其磁性变化相吻合, 表 明电学性质与磁学性质之间存在着相互作用.

\section{补充材料}

本文的补充材料见网络版http://chemcn.scichina.com. 补充材料为作者提供的原始数据, 作者对其学术质量和内容负责.

\section{参考文献}

1 Kumar KS, Ruben M. Coord Chem Rev, 2017, 346: 176-205

2 Bousseksou A, Molnár G, Salmon L, Nicolazzi W. Chem Soc Rev, 2011, 40: 3313-3335

3 Meng YS, Liu T. Acc Chem Res, 2019, 52: 1369-1379

4 Sato O. Nat Chem, 2016, 8: 644-656

5 Gaspar AB, Ksenofontov V, Seredyuk M, Gütlich P. Coord Chem Rev, 2005, 249: 2661-2676

6 Nan CW. Sci Sin-Technol, 2015, 45: 339-357 (in Chinese) [南策文. 中国科学: 技术科学, 2015, 45: 339-357]

7 Scott HS, Staniland RW, Kruger PE. Coord Chem Rev, 2018, 362: 24-43

8 Ge JY, Chen Z, Zhang L, Liang X, Su J, Kurmoo M, Zuo JL. Angew Chem Int Ed, 2019, 58: 8789-8793

9 Yuan J, Wu SQ, Liu MJ, Sato O, Kou HZ. J Am Chem Soc, 2018, 140: 9426-9433

10 Harding DJ, Harding P, Phonsri W. Coord Chem Rev, 2016, 313: 38-61

11 Gütlich P, Goodwin HA. Spin Crossover in transition Metal Compounds I-III. Berlin/Heidelberg: Springer, 2004

12 Halcrow MA. Spin Crossover Materials. Chichester: Wiley, 2013

13 Bauer W, Schlamp S, Weber B. Chem Commun, 2012, 48: 10222-10224

14 Wang HY, Ge JY, Hua C, Jiao CQ, Wu Y, Leong CF, D’Alessandro DM, Liu T, Zuo JL. Angew Chem Int Ed, 2017, 56: 5465-5470

15 Lochenie C, Schötz K, Panzer F, Kurz H, Maier B, Puchtler F, Agarwal S, Köhler A, Weber B. J Am Chem Soc, 2018 , 140: 700-709

16 Liu W, Peng YY, Wu SG, Chen YC, Hoque MN, Ni ZP, Chen XM, Tong ML. Angew Chem Int Ed, 2017, 56: 14982-14986

17 Zhang CJ, Lian KT, Wu SG, Liu Y, Huang GZ, Ni ZP, Tong ML. Inorg Chem Front, 2020, 7: 911-917

18 Jäger EG, Häussler E, Rudolph M, Schneider A. Z Anorg Allg Chem, 1985, 525: 67-85

19 Bentiss F, Lagrenée M, Barbry D. Tetrahedron Lett, 2000, 41: 1539-1541

20 Gómez V, de Pipaón CS, Maldonado-Illescas P, Waerenborgh JC, Martin E, Benet-Buchholz J, Galán-Mascarós JR. J Am Chem Soc, 2015, 137: $11924-11927$

21 Rosario-Amorin D, Dechambenoit P, Bentaleb A, Rouzières M, Mathonière C, Clérac R. J Am Chem Soc, 2018, 140: 98-101

Nihei M, Yanai Y, Hsu IJ, Sekine Y, Oshio H. Angew Chem Int Ed, 2017, 56: 591-594 


\title{
Spin-crossover iron(II) coordination polymers with tetradentate Schiff-base ligands
}

\author{
Ya-Ru Qiu ${ }^{1}$, Jing-Yuan $\mathrm{Ge}^{2}$, Jia-Qian $\mathrm{Li}^{1}$, Jian $\mathrm{Su}^{1 *}$, Jing-Lin Zuo ${ }^{1 *}$ \\ ${ }^{1}$ State Key Laboratory of Coordination Chemistry, School of Chemistry and Chemical Engineering, Nanjing University, Nanjing 210023, China \\ ${ }^{2}$ College of Chemistry \& Materials Engineering, Wenzhou University, Wenzhou 325035, China \\ ${ }^{*}$ Corresponding author (email: zuojl@nju.edu.cn)
}

\begin{abstract}
A series of iron(II) coordination polymers, named [(Fe $\left.\left.\mathrm{Fe}^{\mathrm{II}}\right)\left(\mathrm{L}_{1}\right)(4-\mathrm{abpt})\right](\mathbf{1}),\left[\left(\mathrm{Fe}^{\mathrm{II}}\right)\left(\mathrm{L}_{2}\right)(4-\mathrm{abpt})\right](\mathbf{2})$, $\left[\left(\mathrm{Fe}^{\mathrm{II}}\right)_{2}\left(\mathrm{~L}_{3}\right)_{2}(4-\mathrm{abpt}) \cdot \mathrm{MeOH}\right] \cdot\left[\left(\mathrm{Fe}^{\mathrm{II}}\right)_{2}\left(\mathrm{~L}_{3}\right)_{2}(4-\mathrm{abpt})\right](\mathbf{3})$ and $\left[\left(\mathrm{Fe}^{\mathrm{II}}\right)_{3}\left(\mathrm{~L}_{4}\right)_{3}(4-\mathrm{abpt})_{2} \cdot \mathrm{H}_{2} \mathrm{O}\right](\mathbf{4})\left(\mathrm{H}_{2} \mathrm{~L}_{1}=3,3^{\prime}-((1,2\right.$-phenylenebis (azanediyl))bis(methanylylidene))bis(pentane-2,4-dione), $\mathrm{H}_{2} \mathrm{~L}_{2}=$ diethyl 2,2'-((naphthalene-2,3-diylbis(azanediyl))bis (methanylylidene) $)\left(2 E, 2^{\prime} E\right)$-bis(3-oxobutanoate), $\mathrm{H}_{2} \mathrm{~L}_{3}=$ diethyl 2,2'-((1,2-phenylenebis(azanediyl))bis(methanylylidene))(2E,2'E)-bis(3-oxobutanoate), $\mathrm{H}_{2} \mathrm{~L}_{4}=$ diethyl 2,2'-(((4,5-difluoro-1,2-phenylene)bis(azanediyl))bis(methanylylidene))(2E,2'E)-bis(3-oxobutanoate)), with tetradentate Schiff-base ligands and 3,5-di(pyridin-4-yl)-4H-1,2,4-triazol-4amine (4-abpt) as a bridging ligand were obtained by solvent evaporation method and characterized by elemental analysis, X-ray powder diffraction, magnetic measurements, electrical resistivity and single-crystal X-ray diffraction. Compound 1-4 are one-dimensional (1D) chains. Magnetic measurements reveal that $\mathbf{3}$ posseses a one-step spin transition $\left(T_{1 / 2}=115 \mathrm{~K}\right)$, and compound 4 features two-step spin transitions ( $T_{1 / 2}=188$ and $95 \mathrm{~K}$, respectively) at higher temperatures.
\end{abstract}

Keywords: Fe(II) coordination polymer, spin crossover, crystal structure, Schiff-base ligand

doi: $10.1360 /$ SSC-2020-0128 